\title{
Simultaneous Determination of Six Preservatives in Beverage by HPLC
}

\author{
Shu-Xiang Sun ${ }^{1}$, Shu-Bai $\mathrm{Li}^{2 \mathrm{a}}$ and Pei Yao ${ }^{2}$ \\ 1 Henan Vocational College of Chemical Technology, Zhengzhou, PR China \\ 2 Changzhou Vocational Institute of Engineering, Changzhou, PR China \\ asbli@email.czie.net (Corresponding author)
}

Keywords: High performance liquid chromatography (HPLC), Preservatives, Beverage

Abstract In this study, The aim is to establish the determination method for six preservatives of Benzoic acid, Sorbic acid and four para-hydroxybenzoic acid esters (methyl p-hydroxybenzoate, ethyl p-hydroxybenzoate, propyl p-hydroxybenzoate, butyl p-hydroxybenzoate) in beverage by high performance liquid chromatography(HPLC) simultaneously. After protein precipitation, the sample was diluted by water and separated on a $\mathrm{C} 18$ column by a mobile phase consisting of ammonium acetate $(0.02 \mathrm{M}) /$ methanol in gradient elution and detected at the wavelength of $238 \mathrm{~nm}$. The results shows that The recovery of these six preservatives in the method was $93.2 \%$ to $103.8 \%$ with correlation coefficients from 0.9996 to 0.9999 . The detection limit of these six preservatives was between $0.01 \mathrm{~g} / \mathrm{kg}$ and $0.03 \mathrm{~g} / \mathrm{kg}$. The method developed is sensitive, simple, accurate and suitable for the determination of six preservatives in beverage.

\section{Introduction}

Preservatives are food additives that used to keep the food original quality and nutritional value, they can inhibit microbial growth and reproduction, prevent food spoilage and prolong the shelf life. But researches have proved that eating foods containing excessive amount of preservatives may produce adverse effects on human health, therefore, all countries in the world made strict rules on limitation of food preservatives. There are many researcher make some studies about determination of preservatives[1-7].At present, there are some common additives in the beverage, such as benzoic acid, sorbic acid, methylparaben, Ethylparaben, Propylparaben, Butylparaben. There are corresponding national standard detection methods[8-9] for these preservatives, but there is no simultaneous determination method. Therefore, analytical methods that simultaneously determine multi-preservatives are advantageous. The use of HPLC were determined simultaneously in this paper, and the processing method is simple and rapid, short analysis time, high sensitivity.

\section{Materials and Experimental Methods}

Chemicals The concentration of Benzoic acid, Sorbic acid standard solution were $1.000 \mathrm{mg} / \mathrm{ml}$, they were purchased in the national standard material research center of China. All these reference compounds(Methyl 4-hydroxybenzoate, Ethyl 4-hydroxybenzoate, Propyl 4-hydroxybenzoate, Butyl paraben) have a purity of $\geq 98.0 \%$, they were purchased from ZhenXiang Company(Beijing, China). HPLC-grade ammonium acetate and methanol were obtained from Tedia(American International Chemical, Inc. California, USA). Water was purified using a Milli-Q Ultra pure water purification system(Millipore, Bedford, MA, USA). $0.22 \mu \mathrm{m}$ nylon filter was got from Pall Corporation(USA).All other reagents were analytically pure.

Ultra high performance liquid chromatograph and diode array detector were purchased from UPLC Waters Company (USA), Waters Symmetry C18 chromatographic column $(4.6 \times 75 \mathrm{~mm}, 3.5 \mu \mathrm{m})$ was purchased from Waters Company (USA).

Sample Pretreatment Test samples including carbonated beverage, milk beverage and fruit juice beverage were purchased from local supermarkets in Changzhou, P.R. China. The samples of carbonated beverage were degassed for $10 \mathrm{~min}$ in an ultrasonic bath before being diluted by water. 
About $5.00 \mathrm{~g}$ (accurately weighed to $0.01 \mathrm{~g}$ ) of sample(Milk beverage and juice beverage) were individually weighed into a $50 \mathrm{ml}$ colorimetric tube and dissolved with water, and adding the mixed solution of $5 \mathrm{ml}$ potassium ferrocyanide and $5 \mathrm{ml}$ zinc acetate. Finally, the colorimetric tube was constant volume to $50 \mathrm{ml}$ with water. After being shaken and centrifuged, preservatives were collected through a filter with pore size of $0.22 \mu \mathrm{m}$ before injection into the HPLC system.

About $5.00 \mathrm{~g}$ (accurately weighed to $0.01 \mathrm{~g}$ ) of sample(Carbonated beverage) was weighed into a $50 \mathrm{ml}$ colorimetric tube and dissolved with water, the sample was extracted for $15 \mathrm{~min}$ with ultrasonic degassing. Finally, the colorimetric tube was constant volume to $50 \mathrm{ml}$ with water. After being shaken and centrifuged, preservatives were collected through a filter with pore size of $0.22 \mu \mathrm{m}$ before injection into the HPLC system.

HPLC Detection Conditions The detection wavelength was chosen at 238nm; Column temperature was maintained at $30^{\circ} \mathrm{C}$. The injected sample volume was $10 \mu 1$. The mobile phase was a mixture of methanol and $0.02 \mathrm{M}$ ammonium acetate, and a gradient elution method was used. The optimized chromatographic condition was: 0 6min, methanol 10\%; 7 11min, methanol 70\%; $12 \sim 15 \mathrm{~min}$, methanol $10 \%$, return to initial conditions; a 10 min post-run time was used after each analysis. The column was conditioned prior to use by washing with Methanol and deionizes water successively. The flow-rate used was $0.7 \mathrm{ml} / \mathrm{min}$.

\section{Result and Discussion}

Wavelength Selection of HPLC Ultraviolet absorption spectra (Fig. 1) shows that the maximum absorption wavelength of benzoic acid, sorbic acid and Parabens is $223.3 \mathrm{~nm}, 254.0 \mathrm{~nm}$ and $256.4 \mathrm{~nm}$, respectively. We choose $238 \mathrm{~nm}$ for measuring wavelength. The results show that, the group objects separated completely, and got satisfactory absorbance in this wavelength. In addition, the solvent peak and impurity peaks of the sample won't interfere the determination of the components in the added standards.
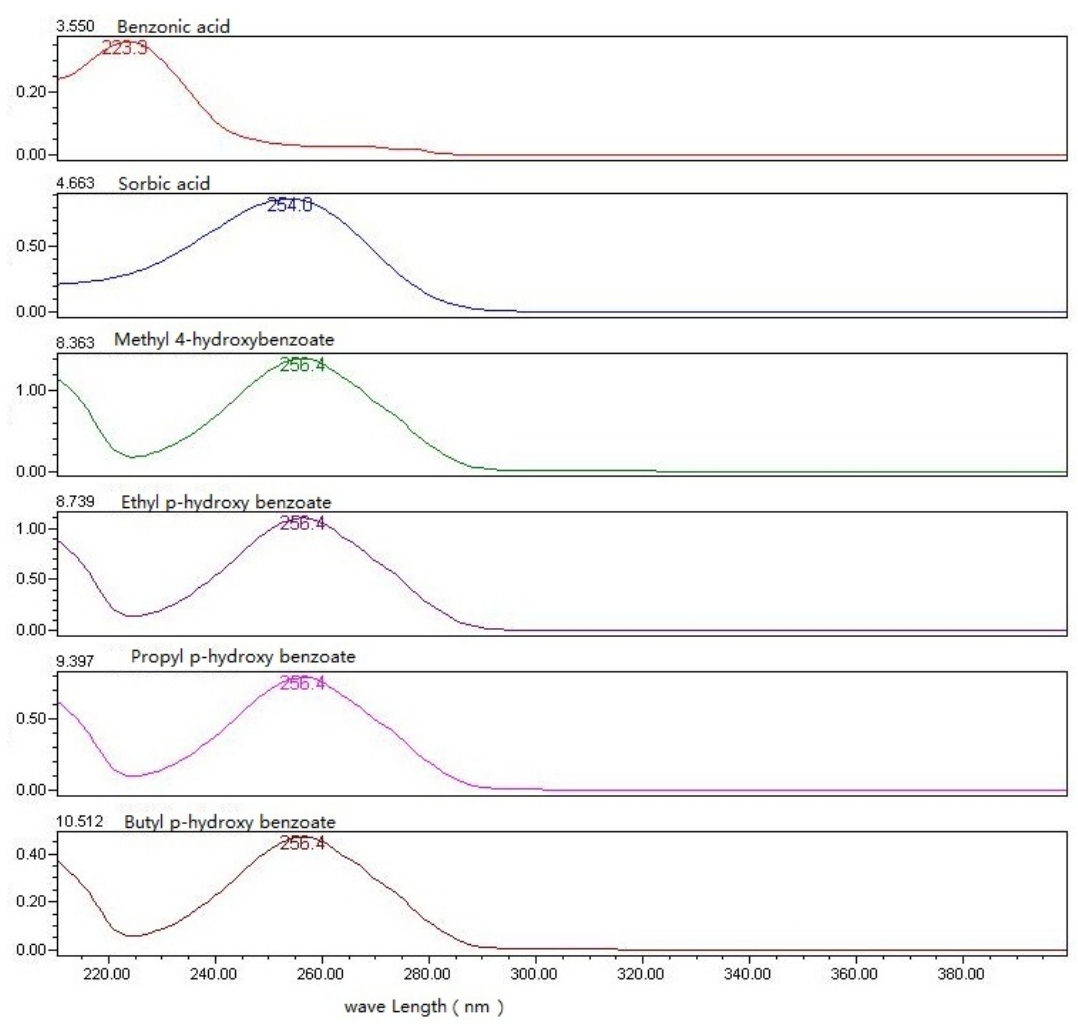

Fig. 1 The UV absorption spectrum of the six preservatives

The Selection of Moving Phase Due to the polarity differences of the benzoic acid, sorbic acid and parabens, they can't be fully eluted off at the right time with the type of isocratic elution. We obtain the satisfactory degree of separation with methanol and $0.02 \mathrm{M}$ ammonium acetate solution as mobile 
phase in a gradient program. The detection can be processed within 15 minutes. Standard spectrum of six preservatives is shown in Fig 2.

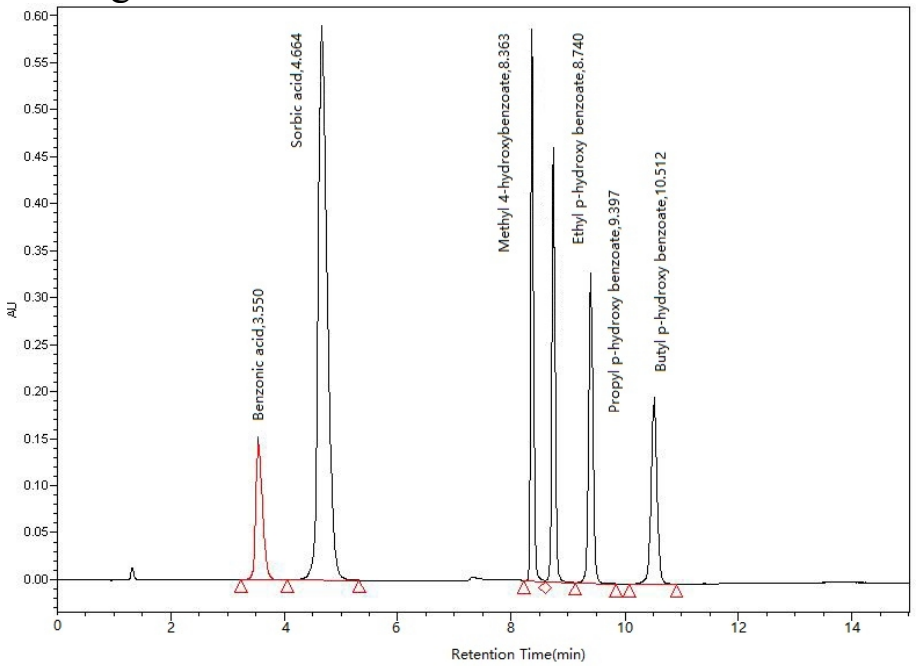

Fig. 2 Standard spectrum of the six preservatives

Calibration Curve To carry out this study, six levels of concentration within the range of $0.01-0.1 \mathrm{mg} / \mathrm{ml}$ working-concentration of the preservatives were prepared. The six preservatives mixed standard solution are prepared in Milli-Q for $0.01 \mathrm{mg} / \mathrm{ml}, 0.02 \mathrm{mg} / \mathrm{ml}, 0.03 \mathrm{mg} / \mathrm{ml}, 0.05 \mathrm{mg} / \mathrm{ml}$ and $0.1 \mathrm{mg} / \mathrm{ml}$ respectively. We determine peak area. The calibration curve was performed with the peak area $\mathrm{Y}$ and concentration of solution $\mathrm{X}(\mathrm{mg} / \mathrm{ml})$, and the equation of linear regression and correlation coefficient $\mathrm{R}$ were obtained. They are listed in Table 1.As observed, the linearity of the analytical response within the studied range of two orders of magnitude is excellent, with correlation coefficients higher than 0.9996 in all case.

Table 1 The equation of linear regression and correlation coefficient of the six preservatives

\begin{tabular}{ccc}
\hline Components name & regression equation & $\mathrm{R}$ \\
\hline benzoic acid & $\mathrm{Y}=2.23 \mathrm{e}+007 \mathrm{X}+3.76 \mathrm{e}+004$ & 0.9996 \\
sorbic acid & $\mathrm{Y}=1.17 \mathrm{e}+008 \mathrm{X}+1.51 \mathrm{e}+005$ & 0.9998 \\
methyl 4-hydroxybenzoate & $\mathrm{Y}=3.67 \mathrm{e}+007 \mathrm{X}+8.15 \mathrm{e}+004$ & 0.9997 \\
ethyl p-hydroxybenzoate & $\mathrm{Y}=3.33 \mathrm{e}+007 \mathrm{X}+9.75 \mathrm{e}+004$ & 0.9999 \\
propyl p-hydroxybenzoate & $\mathrm{Y}=3.12 \mathrm{e}+007 \mathrm{X}+6.84 \mathrm{e}+004$ & 0.9998 \\
butyl p-hydroxybenzoate & $\mathrm{Y}=2.60 \mathrm{e}+007 \mathrm{X}+4.46 \mathrm{e}+004$ & 0.9996 \\
\hline
\end{tabular}

The Sensitivities and Limits of Detection Under the condition of the above methods, we have 3 times the baseline noise determination method detection limit, the detection limit of benzoic acid, sorbic acid, methyl 4-hydroxybenzoate, ethyl p-hydroxybenzoate, propyl p-hydroxybenzoate and butyl p-hydroxybenzoate are $0.01 \mathrm{~g} / \mathrm{Kg}, 0.003 \mathrm{~g} / \mathrm{Kg}, 0.01 \mathrm{~g} / \mathrm{Kg}, 0.01 \mathrm{~g} / \mathrm{Kg}, 0.01 \mathrm{~g} / \mathrm{Kg}$ and $0.01 \mathrm{~g} / \mathrm{Kg}$ respectively.

Recovery Rate and Accuracy In blank samples, add three different concentrations of mixed standard solution respectively, then parallel determination six times, calculate recovery rate and precision. The range of recoveries of benzoic acid, sorbic acid, methyl 4-hydroxybenzoate, ethyl p-hydroxybenzoate, propyl p-hydroxybenzoate and butyl p-hydroxybenzoate were $93.5 \% \sim 102.9 \%$ 、 94.1\% 101.5\%、95.3\% 101.2\%、94.8\% 103.8\%、95.7\% 100.6\%、93.2\% 99.3\% respectively, and the accuracy were $1.03 \% 、 1.22 \% 、 0.92 \% 、 0.64 \% 、 1.35 \% 、 0.86 \%$ respectively.

The Sample Analysis To evaluate the effectiveness of the proposed method, it was applied to the analysis of a total of 30 samples of beverage. Fortunately, in most cases, most preservatives were not found and only benzoic acid was found in some parts of carbonated drinks. And only some sample was found to contain sorbic acid in some parts of fruit juice. P-hydroxy benzoic acid esters were not checked out. 


\section{Conclusion}

A new simple and accurate method has been developed and applied in routine detection preservatives in beverage. This is a real example that illustrates the useful determination for six preservatives with the HPLC simultaneously. The method developed is sensitive, simple, accurate and suitable for the determination of six preservatives in beverage simultaneously. It provides practical technical means to monitor preservatives in beverage. It is important significance in the use of food additives. The method is rapid and has been shown to be reliable.

\section{Acknowledgements}

This work was supported by Colleges and universities in Jiangsu province of outstanding young teachers and principals of overseas training program, Doctoral Foundation of CZIE, and Fund of Jiangsu Student Innovation Training Program.

\section{References}

[1] S. Vidovic', B. Stojanovic', J. Veljkovic', Chromatography A, Vol. 1202 (2008),p.155

[2] N.Xie, T.H. Leng, G.Y. Chen, et al, Chin. J. Analys. Lab. Vol.2(2015),p.222 in Chinese

[3] Y. Zhua, Y.Y. Guo, M.L. Ye, et al. Chromatography A, Vol.1085(2005),p.143

[4] S.F.Song, S.Zhang and D. J. Yang,Chin. J. Health Lab.Techn.Vol.21(2011),p.1369 in Chinese

[5] Q. L.Xiu , Z.G.Feng and Y.S.Yan. Analyt. Chim. Act. Vol.608(2008),p.165

[6] M. D. Paciolla , S. A. Jansen, S. A. Martellucci, et al. J. Pharm. Biom. Analys.Vol.26 (2001),p.143

[7] X.Q. Li, C. Ji, Y.Y. Sun et.al, Food Chem. ,Vol.113 (2009) ,p.692 in Chinese

[8] GB2760-2007. Beijing: Standards Press of China, 2007.

[9] GB/T 23495-2009. Standards Press of China, 2009. 